\title{
Ethnic differences in metabolite signatures and type 2 diabetes: a nested case-control analysis among people of South Asian, African and European origin
}

Irene G. M. van Valkengoed ${ }^{1}$, Carmen Argmann ${ }^{2,3}$, Karen Ghauharali-van der Vlugt ${ }^{2,4}$, Johannes M. F. G. Aerts ${ }^{2,5}$, Lizzy M. Brewster ${ }^{6}$, R. J. G. Peters ${ }^{7}$, Frédéric M. Vaz ${ }^{4}$ and Riekelt H. Houtkooper $\mathbb{B}^{4}$

\begin{abstract}
Accumulation of metabolites may mark or contribute to the development of type 2 diabetes mellitus (T2D), but there is a lack of data from ethnic groups at high risk. We examined sphingolipids, acylcarnitines and amino acids, and their association with T2D in a nested case-control study among 54 South Asian Surinamese, 54 African Surinamese and 44 Dutch in the Netherlands. Plasma metabolites were determined at baseline (2001-2003), and cumulative prevalence and incidence of T2D at follow-up (2011-2012). Weighted linear and logistic regression analyses were used to study associations. The mean level of most sphingolipids was lower, and amino-acid levels higher, in the Surinamese groups than among the Dutch. Surinamese individuals had higher mono- and polyunsaturated acylcarnitines and lower plasma levels of saturated acylcarnitine species than the Dutch. Several sphingolipids and amino acids were associated with T2D. Although only the shorter acylcarnitines seemed associated with prevalent T2D, we found an association of all acylcarnitines (except C0, C18 and C18:2) with incident T2D. Further analyses suggested a potentially different association of several metabolites across ethnic groups. Extension and confirmation of these findings may improve the understanding of ethnic differences and contribute to early detection of increased individual risk.
\end{abstract}

\section{Introduction}

People of South Asian and African origin have a higher type 2 diabetes mellitus (T2D) prevalence and incidence compared with Europeans, at a given body mass index $(\mathrm{BMI})^{1-3}$. Higher tissue and plasma concentrations of metabolites may contribute to the development of T2D. Prominent is the accumulation of lipids that culminates to lipotoxicity. Primary classes of intracellular lipid mediators associated with T2D and insulin resistance include cytosolic lipids such as sphingolipids, and mitochondriaderived metabolites such as acylcarnitines ${ }^{4-7}$. In addition

\footnotetext{
Correspondence: Irene Valkengoed (i.g.vanvalkengoed@amc.uva.nl)

'Department of Public Health, Academic Medical Centre, I. van Valkengoed, Meibergdreef 9, J2-209, 1105 AZ Amsterdam, The Netherlands

${ }^{2}$ Department of Medical Biochemistry, Academic Medical Centre, I. van

Valkengoed, Meibergdreef 9, J2-209, 1105 AZ Amsterdam, The Netherlands

Full list of author information is available at the end of the article
}

to fat metabolites, there is a marked association between amino acids and $\mathrm{T}_{2} \mathrm{D}^{8,9}$. Recent evidence suggests that associations may vary by ethnicity, for example, for amino acids in men ${ }^{9}$. This could signal differences in intake and the relative importance of metabolic processes across groups. However, data are limited. We examined ethnic differences in these metabolites and their association with T2D in people of South Asian, African and European origin in the Netherlands.

\section{Subjects and methods \\ Study population}

We carried out a nested case-control study. In brief, 1444 South Asian Surinamese, African Surinamese and European Dutch (henceforth Dutch) completed an interview and medical examination in the SUNSET study between 2001 and $2003^{1,3}$. In 2011-2012, participants 
who still resided in Amsterdam were invited to take part in a follow-up examination within the HELIUS study ${ }^{1,2}$.

We excluded individuals with missing data on T2D at either measurement or with self-reported baseline cardiovascular disease. From the group that remained, we took a sample from strata characterized by ethnicity, sex and T2D status at follow-up. Data for 54 South Asian Surinamese, 54 African Surinamese and 44 Dutch were available for analysis.

The Institutional Review Board of the Academic Medical Centre approved the studies. Participants provided a written informed consent.

\section{Measurements}

Ethnicity was classified based on self-identification ${ }^{1,3}$. BMI was calculated as weight in kilograms by height in meters squared. The covariate BMI change was calculated by subtracting BMI at follow-up from the baseline BMI. Healthy diet was defined in line with Nicolaou et al. ${ }^{9}$, based on 24 questions about the usual amount and frequency of intake of common food items and items more specific for the Surinamese (e.g., salty condiments). The individual dietary items were combined to form a composite score based on the Dutch guidelines for: fruit (2 points for two pieces of fruit or one piece of fruit and one glass of juice daily (no sugar added); 1 point for one piece of fruit daily or two pieces 5-6 days per week), vegetables ( 2 points for $200 \mathrm{~g}$ daily, 1 point for $150 \mathrm{~g}$ daily or $200 \mathrm{~g}$ 5-6 days per week), salt (1 point for minimal use of salt at cooking and at the table), fish (1 point for at least once a week), red meat (1 point for twice a week or less), breakfast ( 2 points for daily; 1 point for 5-6 days per week), fat in cooking (1 point for mostly oil).

Baseline T2D was defined as a fasting plasma glucose (HK/Glucose-6-P-dehydrogenase test; Roche-Diagnostics) $\geq 7.0 \mathrm{mmol} / \mathrm{l}$ and/or self-reported T2D. At followup, T2D was defined as an $\mathrm{HbA} 1 \mathrm{c} \geq 6.5 \%(48 \mathrm{mmol} / \mathrm{mol})$ and/or a fasting plasma glucose $\geq 7.0 \mathrm{mmol} / \mathrm{l}$ and/or selfreported $\mathrm{T} 2 \mathrm{D}^{1,10}$.

In 2012, we measured metabolite concentrations in baseline plasma samples that had been stored at $-80^{\circ} \mathrm{C}$. The analyses were done as reported previously ${ }^{11,12}$.

\section{Statistical analysis}

We used methods that weighted for the sampling strategy used. Baseline data were expressed as percentages or means with $95 \%$ confidence intervals. Ethnic differences in metabolites were calculated with linear regression, adjusted for the continuous variables age and $\mathrm{BMI}^{1}$; and, additionally, with adjustment for healthy diet score as a continuous variable. Sex was not considered, as it was previously not associated with $\mathrm{T}_{2} \mathrm{D}^{3}$ and had been used as sampling variable. Repeated analyses with non-parametric methods did not change results (data not shown).
Subsequently, we examined the age and BMI adjusted association of the metabolites with cumulative T2D. In this analysis, we included all cases $(n=44)$, including those already identified at baseline. Moreover, we repeated the analysis with adjustment for BMI change and with further adjustment for healthy diet as a continuous variable. We also repeated the analysis after excluding persons with T2D at baseline, with additional adjustment for baseline fasting glucose. Finally, we evaluated differences in the association with cumulative T2D by ethnicity, reporting only report the metabolites with $p \leq 0.10$ for the interaction.

Analyses were performed using the SAS package, version 9.3 (SAS Institute Inc., Cary, NC, USA). No power calculations were performed. We considered a $p \leq 0.05$ indicative of an association (two sided).

\section{Results}

Baseline characteristics differed by ethnicity (Supplement 1). The mean level of most sphingolipids was lower, and plasma amino-acid levels higher, in the Surinamese groups than the Dutch (Table 1; Supplement 2). For baseline acylcarnitine levels, there appears to be an overall switch in saturation, as Surinamese had higher mono- and polyunsaturated acylcarnitines and lower plasma levels of saturated acylcarnitine species. Additional adjustment for diet did not account for these differences (Supplement 3).

Metabolite levels were associated with prevalent T2D (Table 2). Adjustment for diet or BMI change did not affect our interpretation (Supplement 5). The restricted analyses (incident only) also largely confirmed the associations for sphingolipids and amino acids; a limited number of metabolites, for example, alanine and leucine, were no longer associated, whereas others appeared similarly or even more strongly associated (e.g., lactosylceramide, methionine and proline; Table 2). For the acylcarnitines, however, the difference was notable. Although only the shorter acylcarnitines seemed associated with cumulative T2D, we found an association of higher mean baseline levels of all acylcarnitines (except C0, C18 and C18:2) with incident T2D. Further analyses showed some evidence for a differential association of several metabolites with the T2D risk by ethnicity (Supplement 5).

\section{Discussion}

South Asian and African Surinamese had lower sphingolipid, higher plasma amino acid, higher mono- and polyunsaturated acylcarnitine and lower saturated acylcarnitine plasma levels than the Dutch. Differences in these metabolites were associated with cumulative and incident T2D. The observed differences in metabolite levels may reflect the specific dietary intakes of the ethnic groups in the Netherlands ${ }^{2,}{ }^{13}$. However, the available 


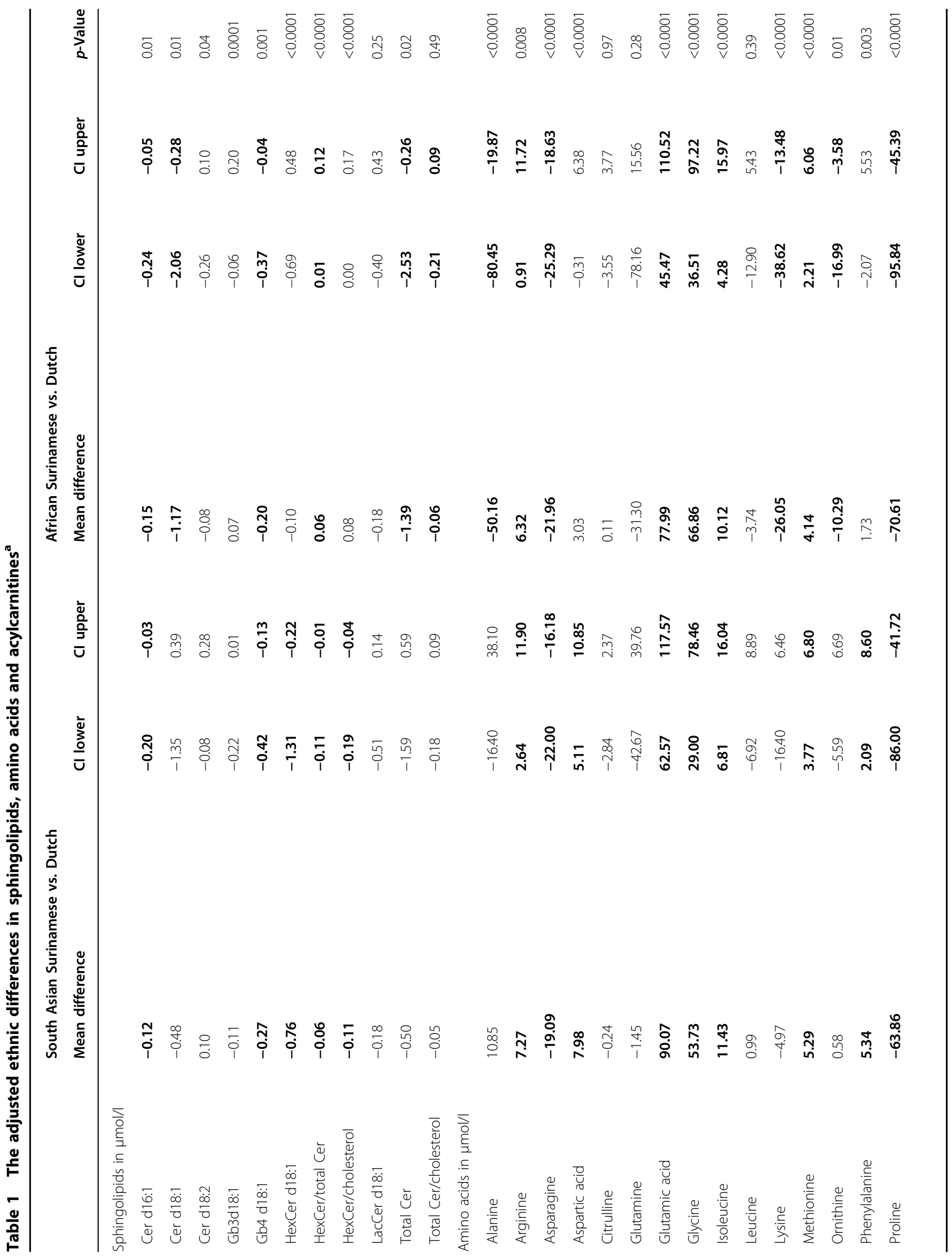




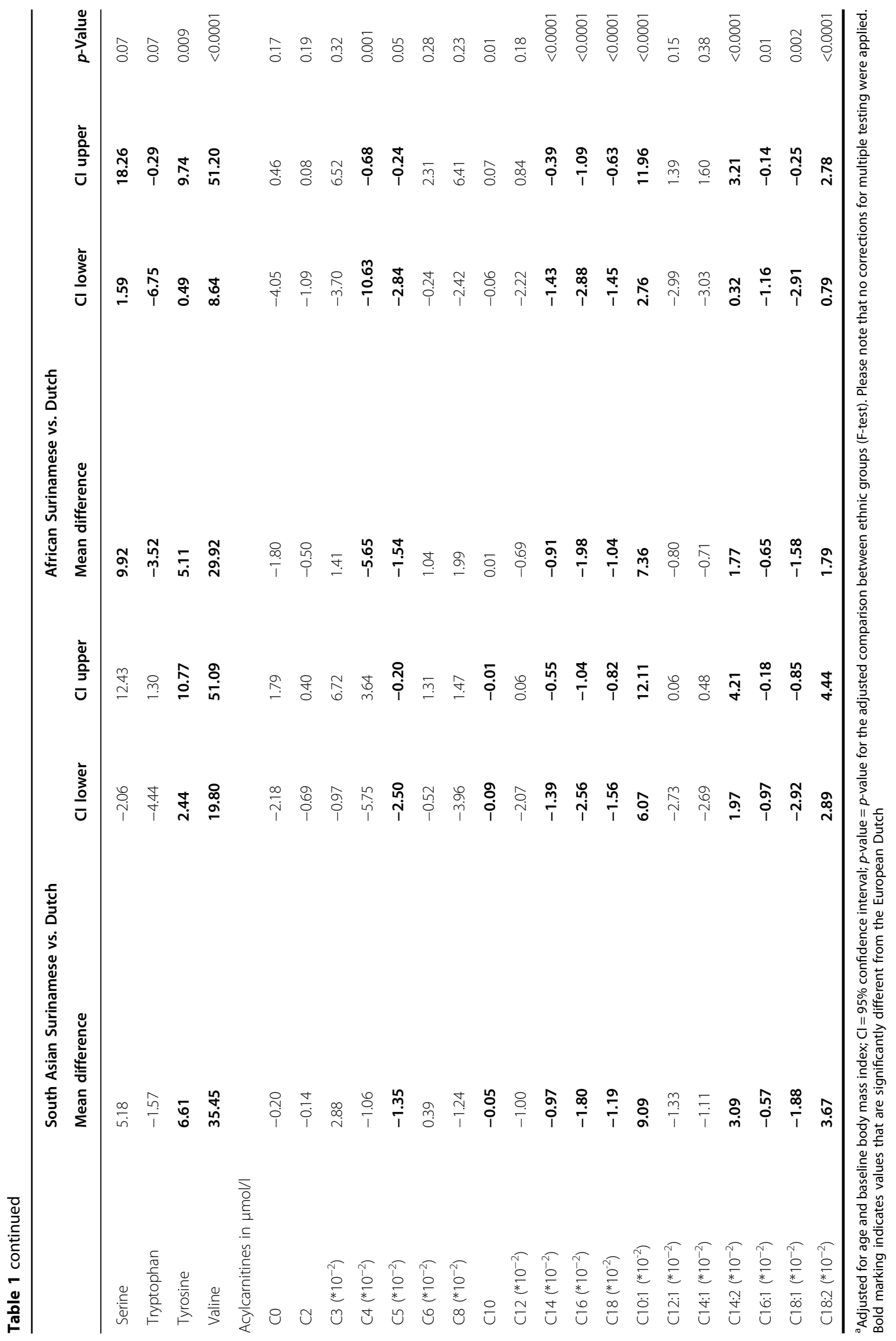




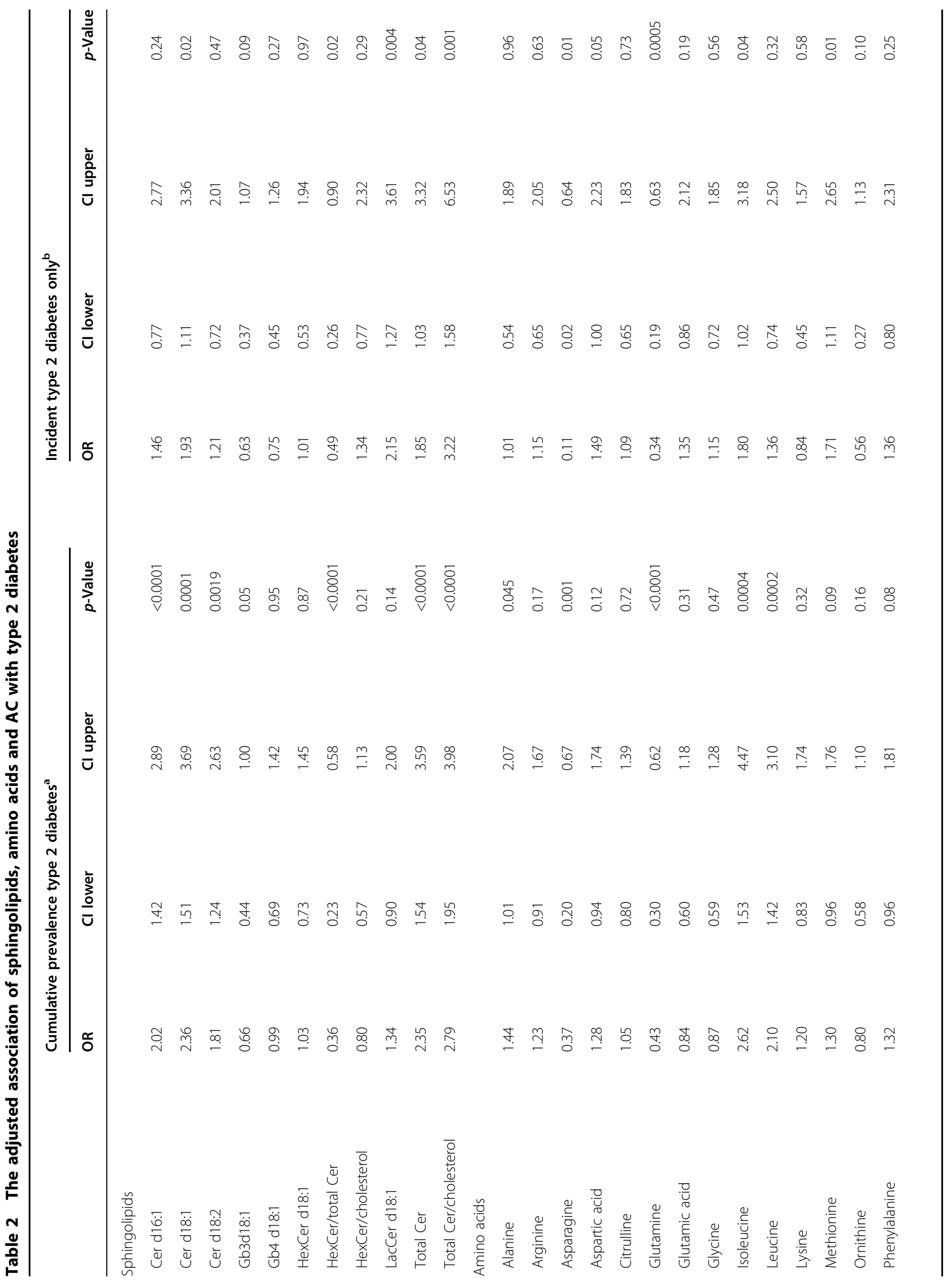




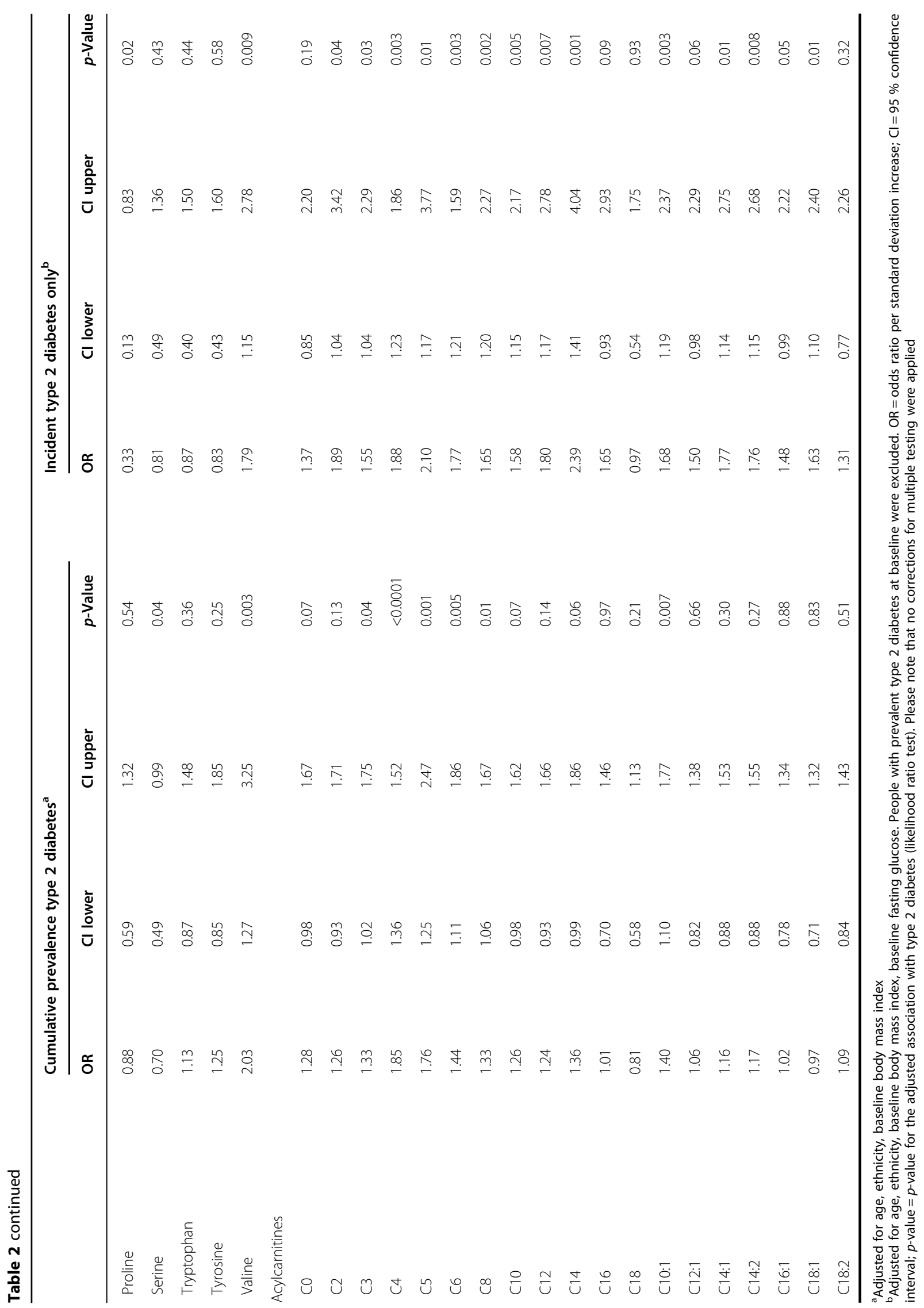


self-reported data on diet did not explain associations in the current study. In addition, differences may reflect ethnic differences in (nutrient) metabolism. A difference in brown adipose tissue volume and capacity in South Asians has been found that may contribute to an reduced energy expenditure and T2D risk ${ }^{14}$.

We found that higher levels of several sphingolipids, for example, ceramide and lactosylceramide, were associated with T2D, with evidence for a somewhat stronger association among those of African origin than others. To the best of our knowledge, no data have been reported for Africans or South Asians. However, the association of ceramide is consistent with the reported association in Australians and Mexican Americans ${ }^{6}$.

The association of acylcarnitines with T2D also fits reported associations of several, in particular short and medium chain, acylcarnitines with prevalent T2D in obese African-American women ${ }^{5}$. However, another study did not find strong associations with insulin resistance ${ }^{4}$. Differences in the association between African, South Asian and European origin populations were not reported.

The associations of T2D with the three branched-chain amino acids (BCAAs) and to a lesser extent phenylalanine, as well as the lack of association with, for example, ornithine, citrulline and arginine, are mostly in line with previous work ${ }^{4,7}$. However, a recent study did not find an association of BCAA in South Asian family members of people with $\mathrm{T} 2 \mathrm{D}^{15}$. The association of tyrosine was consistent with one study, and inconsistent with another ${ }^{4,7}$. Finally, our study suggested some associations with other amino acids, for example, the negative association with serine and proline, which were not found previously ${ }^{4}$.

Ethnic differences in associations with incident T2D, for instance for BCAA and tyrosine, have been reported ${ }^{7}$. Interestingly, these differences were not significant in our study, but we found some evidence for a possibly different association of other amino acids with cumulative T2D. Yet, the numbers are too small to draw firm conclusions.

If confirmed, our findings may have implications for the use of metabolites to enhance risk assessment for incident diabetes ${ }^{16}$. Further exploration of ethnic differences may also point toward new pathways underlying the excess burden of T2D in some groups.

\section{Limitations}

Our observational data have several limitations. First, causality cannot be inferred. Second, we determined metabolite levels in stored plasma samples, which may better reflect levels in certain tissues and organs than others ${ }^{17}$. Reported levels may not be reflective of all (changes in) relevant metabolic processes.

Third, our analysis was based on cumulative cases at follow-up. Results of the analysis of incident cases largely confirmed the associations, but the power was limited and we could not explore all potential confounders and interactions.

Finally, in line with previous work $^{9}$, we chose not to apply a correction for multiple testing. Although this may imply some false-positive results, even a strict Bonferroni correction would not have explained all associations.

In conclusion, we found large ethnic differences in sphingolipids, acylcarnitines and amino acids, which were associated with T2D in people of South Asian, African and European origin. Extension and confirmation of these findings may improve the understanding of ethnic differences in T2D and contribute to early detection of increased individual risk.

\section{Acknowledgements}

We thank W. Busschers (statistics), and H. Rusch and A. van Cruchten (metabolite analyses). No specific funding was received for this work.

\section{Author details}

${ }^{1}$ Department of Public Health, Academic Medical Centre, I. van Valkengoed, Meibergdreef 9, J2-209, 1105 AZ Amsterdam, The Netherlands. Department of Medical Biochemistry, Academic Medical Centre, I. van Valkengoed,

Meibergdreef 9, J2-209, 1105 AZ Amsterdam, The Netherlands. ${ }^{3}$ Department of Genetics and Genomic Sciences and Icahn Institute for Genomics and

Multiscale Biology, Icahn School of Medicine at Mount Sinai, New York, NY, USA. ${ }^{4}$ Laboratory Genetic Metabolic Diseases, Academic Medical Centre, I. van Valkengoed, Meibergdreef 9, J2-209, 1105 AZ Amsterdam, The Netherlands. ${ }^{5}$ Department of Biochemistry, Leiden Institute of Chemistry, Leiden, The Netherlands. ${ }^{6}$ Department of Vascular Medicine, Academic Medical Centre, I. van Valkengoed, Meibergdreef 9, J2-209, 1105 AZ Amsterdam, The Netherlands. 'Department of Cardiology, Academic Medical Centre, I. van Valkengoed, Meibergdreef 9, J2-209, 1105 AZ Amsterdam, The Netherlands

\section{Competing interests}

The authors declare that they have no competing financial interests.

\section{Publisher's note}

Springer Nature remains neutral with regard to jurisdictional claims in published maps and institutional affiliations.

\section{Supplementary information}

The online version of this article (https://doi.org/10.1038/s41387-017-0003-z) contains supplementary material, which is available to authorized users.

Received: 12 December 2016 Revised: 6 September 2017 Accepted: 17 September 2017

Published online: 13 December 2017

\section{References}

1. Admiraal, W. M. et al. Ethnic disparities in the association of impaired fasting glucose with the 10-year cumulative incidence of type 2 diabetes. Diabetes Res. Clin. Pract. 103, 127-132 (2014).

2. Dekker, L. H. et al. Comparable dietary patterns describe dietary behavior across ethnic groups in the Netherlands, but different elements in the diet are associated with glycated hemoglobin and fasting glucose concentrations. J. Nutr. 145, 1884-1891 (2015).

3. Bindraban, N. R. et al. Prevalence of diabetes mellitus and the performance of a risk score among Hindustani Surinamese, African Surinamese and ethnic Dutch: a cross-sectional population-based study. BMC Public Health 8, 271 (2008).

4. Palmer, N. D. et al. Metabolomic profile associated with insulin resistance and conversion to diabetes in the Insulin Resistance Atherosclerosis Study. J. Clin. Endocrinol. Metab. 100, E463-E468 (2015). 
5. Adams, S. H. et al. Plasma acylcarnitine profiles suggest incomplete long-chain fatty acid beta-oxidation and altered tricarboxylic acid cycle activity in type 2 diabetic African-American women. J. Nutr. 139(6), 1073-1081 (2009)

6. Meikle, P. J. et al. Plasma lipid profiling shows similar associations with prediabetes and type 2 diabetes. PLOS ONE 27(8), e74341 (2013).

7. Holland, W. L. \& Summers, S. A. Sphingolipids, insulin resistance, and metabolic disease: new insights from in vivo manipulation of sphingolipid metabolism. Endocr. Rev. 29, 381-402 (2008)

8. Wang, T. J. et al. Metabolite profiles and the risk of developing diabetes. Nat Med. 17, 448-453 (2011)

9. Tillin, T. et al. Diabetes risk and amino acid profiles: cross-sectional and prospective analyses of ethnicity, amino acids and diabetes in a South Asian and European cohort from the SABRE (Southall And Brent REvisited) Study. Diabetologia 58, 968-979 (2015).

10. American Diabetes Association. Classification and diagnosis of diabetes. Diabetes Care 38(Supplement 1), S8-S16 (2015).

11. Groener, J. E. et al. HPLC for simultaneous quantification of total ceramide glucosylceramide, and ceramide trihexoside concentrations in plasma. Clin. Chem. 53, 742-747 (2007 Apr).
12. Houtkooper, R. H. et al The metabolic footprint of aging in mice. Sci. Rep. 1, 134 (2011).

13. Nicolaou, M., van Dam, R. M. \& Stronks, K. Acculturation and education level in relation to quality of the diet: a study of Surinamese South Asian and AfroCaribbean residents of the Netherlands. J. Hum. Nutr. Diet. 19, 383-393 (2006).

14. Bakker, L. E. et al. Brown adipose tissue volume in healthy lean south Asian adults compared with white Caucasians: a prospective, case-controlled observational study. Lancet Diabetes Endocrinol. 2, 210-217 (2014).

15. Jainandunsing, $\mathrm{S}$. et al. Discriminative ability of plasma branched-chain amino acid levels for glucose intolerance in families at risk for type 2 diabetes. Metab. Syndr. Relat. Disord. 14, 175-181 (2016).

16. Sun, L. et al. Early prediction of developing type 2 diabetes by plasma acylcarnitines: a population-based study. Diabetes Care 39, 1563-1570 (2016).

17. Schooneman, M. G. et al. Transorgan fluxes in a porcine model reveal a central role for liver in acylcarnitine metabolism. Am. J. Physiol. Endocrinol. Metab. 309 E256-E264 (2015). 